\title{
Refinement of the GINGF3 locus for hereditary gingival fibromatosis
}

\author{
Michael Pampel • Sandra Maier • Alfons Kreczy • \\ Helga Weirich-Schwaiger • Gerd Utermann • \\ Andreas R. Janecke
}

Received: 26 January 2009 / Accepted: 8 July 2009 / Published online: 26 July 2009

(C) The Author(s) 2009. This article is published with open access at Springerlink.com

\begin{abstract}
Hereditary gingival fibromatosis (HGF) is a rare, clinically variable disorder characterized by slowly progressive fibrous overgrowth of the gingiva. Four gene loci have been mapped for autosomal dominant non-syndromic HGF (adHGF). The molecular basis of adHGF remains largely unknown, with only a single SOS1 gene mutation identified so far at the gingival fibromatosis 1 (GINGF1) locus in one family. We identified an adHGF family with ten affected individuals in whom onset of gingival fibromatosis concurred with the eruption of the primary teeth. In order to identify the molecular basis in this family, we tested for linkage of the disease to known adHGF loci. A maximal multipoint logarithm of the odds score of 3.91 was obtained with marker D2S390 $(\theta=0)$ at the GINGF3 locus on chromosome 2p23.3-p22.3, and linkage to other known loci was excluded. Sequencing two candidate genes, $A L K$ and $C 2$ orf 18, and a single nucleotide polymorphisms array analysis did not reveal a mutation or copy number variation in a patient from the family. We refined the GINGF3 locus to a $6.56-\mathrm{cM}, 8.27-\mathrm{Mb}$ region containing 112 known and hypothetical genes, and our data and a
\end{abstract}

M. Pampel

Coburg, Germany

S. Maier $\cdot$ H. Weirich-Schwaiger $\cdot$ G. Utermann $\cdot$

A. R. Janecke $(\bowtie)$

Division of Clinical Genetics, Innsbruck Medical University,

Schoepfstrasse 41,

6020 Innsbruck, Austria

e-mail: Andreas.Janecke@i-med.ac.at

A. Kreczy

Institute of Pathology, Hospital Coburg,

Coburg, Germany search of the literature suggest that GINGF3 is a major adHGF locus.

Keywords Nodular - Alveolar · Signaling - ALK gene · C2orf1 8 - Major gene · Primary teeth · Gingiva - SNP - Array · Copy number analysis

\section{Introduction}

Gingival fibromatosis (gingival hyperplasia) may result from systemic medication with calcium-channel blockers, cyclosporin, dilantin, and phenytoin, or it may be hereditary. In severe cases, the gingival enlargement may cover the crowns of teeth and cause severe functional and esthetic concerns. Hereditary gingival fibromatosis (HGF) is a rare condition that can occur as an isolated disease or as part of a number of syndromes or chromosomal abnormalities [1, 2]. The onset of gingival overgrowth generally coincides with the eruption of the permanent incisors, infrequently with the eruption of the primary dentition, and it rarely presents already at birth. Among the non-syndromic HGFs, autosomal recessive [3] and autosomal dominant inheritance have been described [2]. Linkage studies have localized loci for autosomal dominant non-syndromic forms of gingival fibromatosis (adHGF) to chromosomes 2p21p22 (GINGF1, OMIM 135300) [4, 5], 2p22.3-p23.3 (GINGF3, OMIM 609955) [6], and 5q13-q22 (GINGF2, OMIM 605544) [7]. Delineation of chromosome 2p13-p21 duplication in a patient with HGF in association with mental retardation and facial dysmorphism suggested another HGF locus on chromosome 2p13-p21 [8]. Recently, an apparently maternally inherited form of gingival fibromatosis was mapped to chromosome $11 \mathrm{p} 15$ in two unrelated Chinese families [9]. The molecular basis of HGF 
remains largely unknown. One mutation in the SOS1 (son of sevenless one) gene (OMIM 182530) has been identified at the GINGF1 locus in a single adHGF family. Diagnosis of HGF is based on medical history and clinical examination, since there are currently no specific immunohistochemical markers available.

\section{Materials and methods}

We ascertained a German four-generation family with autosomal dominant transmission of HGF without evidence for parental imprinting through a proband. Fifteen family members were examined and nine (five male and four female) were classified as affected (Fig. 1, individuals $12170,3,11284,13230,11602,12163,13232,11603$, and 11606) based on the following criteria: enlarged gingiva covering at least one third of clinical dental crowns of five or more teeth and lack of exposure of affected members to any inducible drugs; individuals were otherwise healthy. The deceased male founder of this family (Fig. 1, individual 1) was reported as having been affected by HGF. Five spouses of family members were also examined, and samples were taken for molecular investigation (individuals 12021, 11378, 13231, 11253, and 11760 in Fig. 1). No samples were available from individuals 1-6 shown in Fig. 1, and their genotypes were inferred and haplotypes were re-constructed (see below).

Informed consent was obtained from all participants following genetic counseling. DNA was extracted from peripheral blood samples, from paraffin-embedded tissue sections, and from buccal smears, and RNA was extracted from cultured fibroblasts using an automated extractor according to the manufacturer's protocols (GenoM 48, Qiagen, Vienna, Austria). For linkage analysis, highly polymorphic microsatellite markers were selected from 2p21-p23.3 and from 5q13-q22 (http://www.gdb.org/). Locus order and sex-averaged inter-marker distances were taken from the Marshfield map (http://research.marshfield clinic.org/genetics/). Marker alleles were detected by electrophoresing the PCR products on $6 \%$ polyacrylamide gels followed by silver staining. We used the Allegro program [10] to compute haplotypes as well as two-point and multipoint logarithm of the odds (LOD) scores, under the assumption of autosomal dominant inheritance with $100 \%$ penetrance, and with the disease-allele frequency set at.0001, and equal marker allele frequencies. A whole-genome analysis of copy number variation was conducted in one patient (12170, Fig. 1) using the Affymetrix Genome-Wide Human SNP 6.0 array according to the specifications of the manufacturer. Oligonucleotide primer sequences and conditions to sequence the ALK and C2orf18 genes in complementary DNA (cDNA) are available on request.

\section{Results}

Remarkably, the onset of gingival fibromatosis consistently concurred with the eruption of the primary teeth in all affected members of this family. Gingival fibromatosis developed slowly progressive and variably consisted of localized or generalized enlargement of keratinized gingiva and consisted of dense fibrous tissue that feels firm and nodular on palpation (Fig. 2a-d). Gingival excess tissue resulted in periodontal problems. Difficulties in daily oral hygiene and long-term smoking were recorded in patients with most severe findings (Fig. 2c, d). Histological evaluation of specimens from different individuals of the family showed hyperplasia of fibrous tissue characterized by squamous epithelium with elongated rete ridges overlaying dramatically increased cell poor fibrous tissue (Fig. 3). A pseudoepitheliomatous hyperplasia of the squamous epithelium with formation of papillae was seen in severely affected individuals.

Haplotype and LOD score analyses excluded GINGF1 (Figs. 1 and 4 and Table 1) and GINGF2 (data not shown) in this adHGF family. Linkage of the disease to GINGF3 was demonstrated with a maximal two-point LOD score of 3.45 with marker $D 2 S 171(\theta=0$; Table 1$)$ and a maximal multipoint LOD score of 3.91 with marker D2S390 $(\theta=0$; Fig. 4). Recombinations defined markers $D 2 S 220$ and D2S352 as boundaries of the linked interval (Fig. 1). The candidate region defined in the presented family overlaps with the 11.42-cM, 13.04-Mb GINGF3 interval flanked by marker loci D2S2221 and D2S1788, originally defined in one Chinese family [6]. According to the current NCBI draft sequence of the human genome, build 36.3, the original and the refined GINGF3 candidate region contain 131 and 112 known and hypothetical genes. Prioritarization of candidate genes for mutation analysis considers expression in gingival or connective tissue and a putative function of the gene product in cell cycle control or extracellular matrix composition. Three interactive, web-based implementations, SUSPECTS (http://www. genetics.med.ed.ac.uk/suspects/), GeneWanderer (http:// compbio.charite.de/genewanderer/GeneWanderer), and GeneDistiller (http://www.genedisteller.org) [11] were queried in addition to a manual approach to identify the disease gene based on expression and functional data contained in the NCBI database (http://www.ncbi.nlm.nih. gov/sites/gene). However, sequencing two candidate genes, $A L K$, encoding a receptor protein-tyrosine kinase and $C 2$ orf 18 encoding an adenine nucleotide translocase 2 binding protein in cDNA from fibroblasts did not reveal a mutation in a patient from the presented family. A wholegenome analysis of copy number variation in the same patient detected neither aberrations at the GINGF3 locus nor elsewhere. 


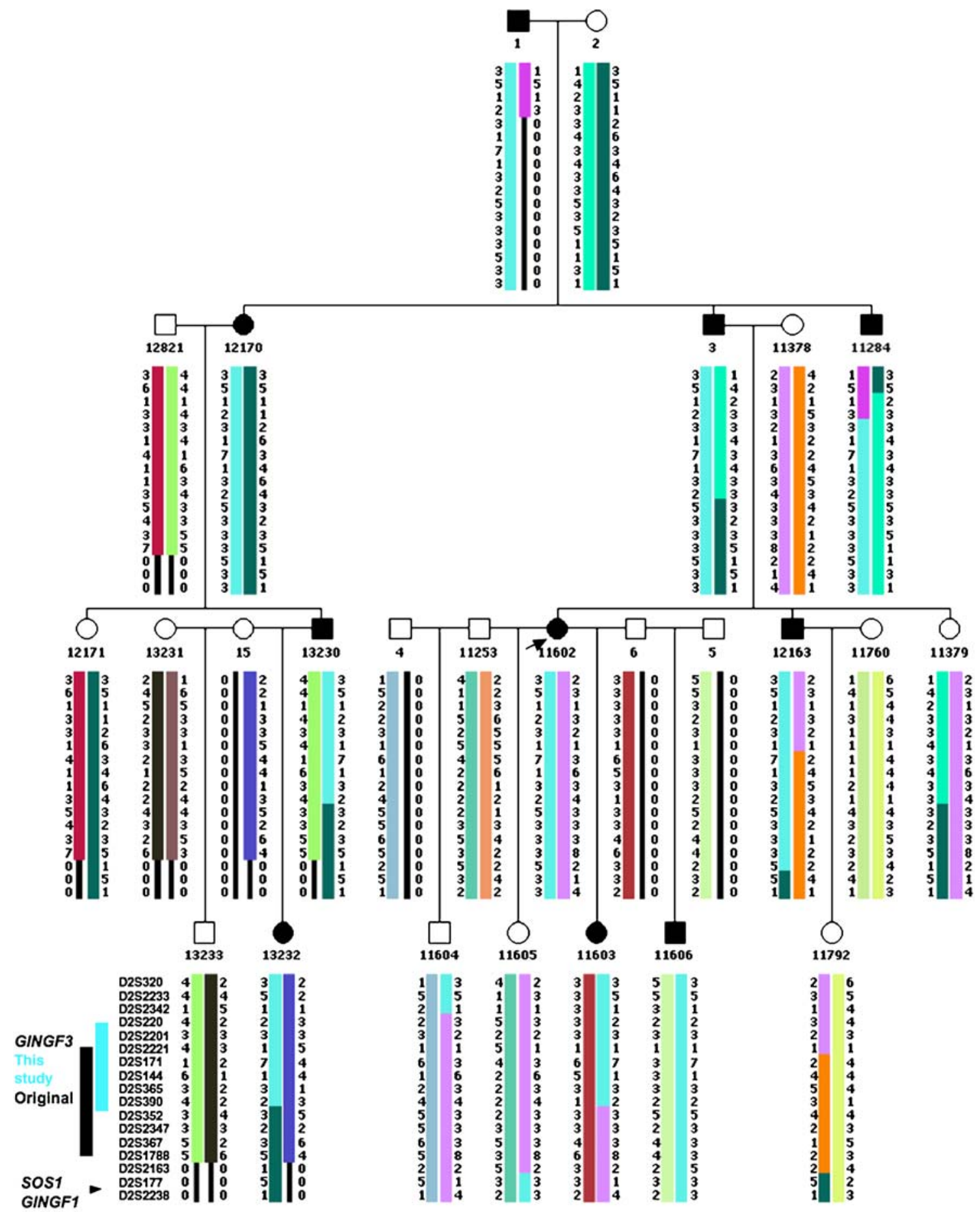

Fig. 1 Pedigree and haplotypes of the HGF family under study. The proband, indicated by an arrow, has two affected and two unaffected children with four different partners. Haplotype analysis excluded the SOS1 gene at the GINGF1 locus as the disease gene in this family and

\section{Discussion}

The candidate region defined in the presented family overlaps with the GINGF3 interval originally defined in one Chinese family [6]. Under the hypothesis that this locus narrowed the original 11.42-cM, 13.04-Mb GINGF3 locus, flanked by marker loci D2S2221 and D2S1788, down to the region between markers D2S220 and D2S352

contains a single HGF gene, we refined it to a $6.56-\mathrm{cM}$, $8.28-\mathrm{Mb}$ interval flanked by maker loci $D 2 S 2221$ and D2S352 (Figs. 1, 4) and confirm the presence of an additional HGF gene on chromosome $2 p$ distinct from the GINGF1 locus. The data of at least five published smaller 
Fig. 2 Gingival overgrowth in a a 6-year-old female and $\mathbf{b}$ 13year-old male showing symmetrical gingival hyperplasia.

c Severe involvement in a male at age 36 years and $\mathbf{d}$ a female proband aged 37 years, 7 years after last surgical intervention
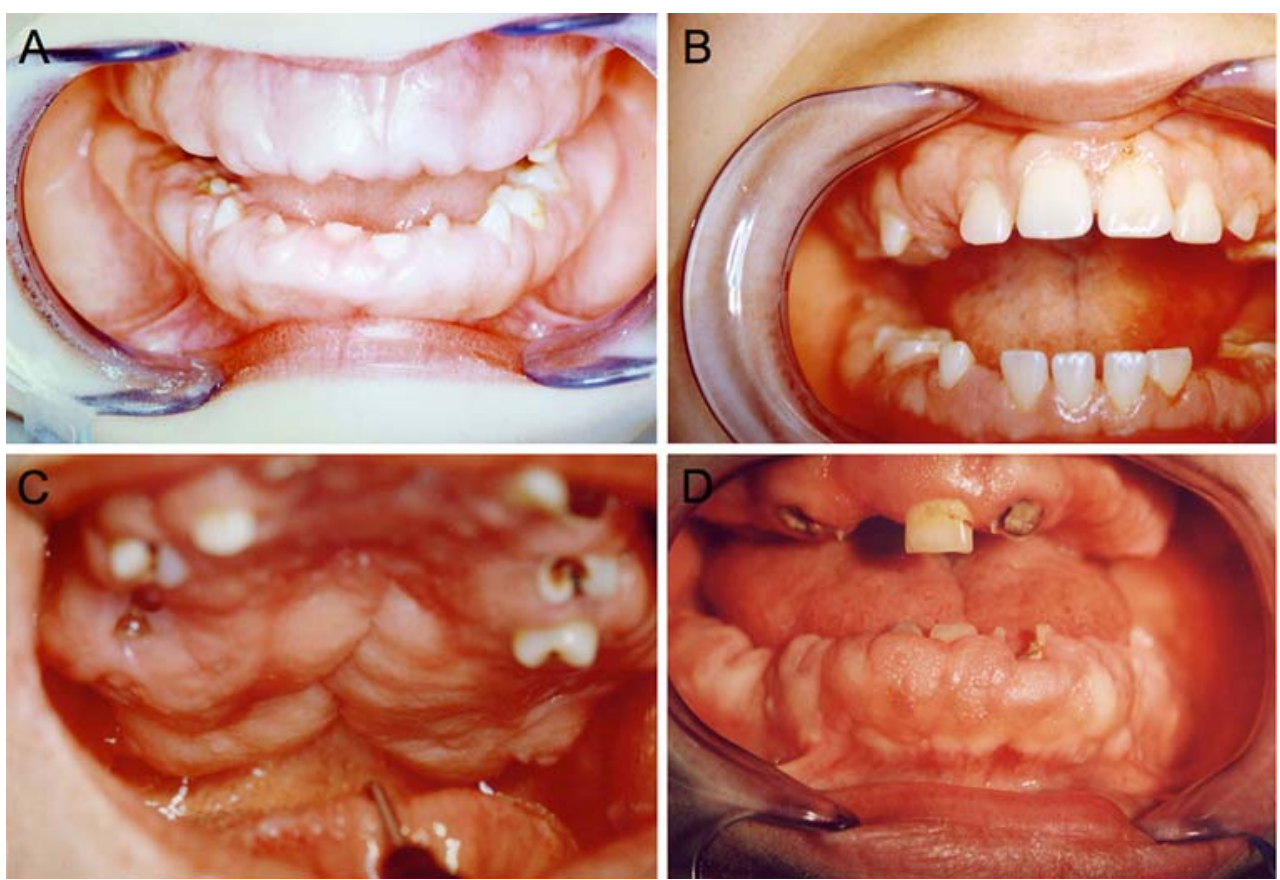

adHGF families are compatible with linkage to this locus $[5,6]$. These previous reports and our data suggest that GINGF3 is a major adHGF locus.

Previous studies on HGF suggest a function of the GINGF3 gene product in cell cycle control, and more specifically, the gene product might directly or indirectly interact with SOS1. A heterozygous SOS1 single base insertion leading to a C-terminally truncated protein, leading to sustained activation of RAS/MAPK signaling in fibroblasts, was identified in the form of $\operatorname{HGF}[12,13]$. Mutations in another component of the RAS/MAPK pathway, HRAS, can cause Costello syndrome, a malformation-dysmorphism syndrome with gingival hypertrophy as a feature [14]. Most recently, dysregulation of the human mitogen-activated protein kinase kinase 6 (MAP2K6) was implicated in the pathogenesis of a syndromic form of HGF, congenital generalized hypertrichosis terminalis [15]. Therefore, genes from the candi-
Fig. 3 Histopathological findings in two individuals from the presented family. a Squamous epithelium with elongated rete ridges overlaying dramatically increased cell poor fibrous tissue (original magnification, $\times 100$, H\&E stain). b Pseudoepitheliomatous hyperplasia of the squamous epithelium with formation of papillae in individuals with marked inflammatory infiltrates (original magnification, $\times 100, H \& E$ stain)
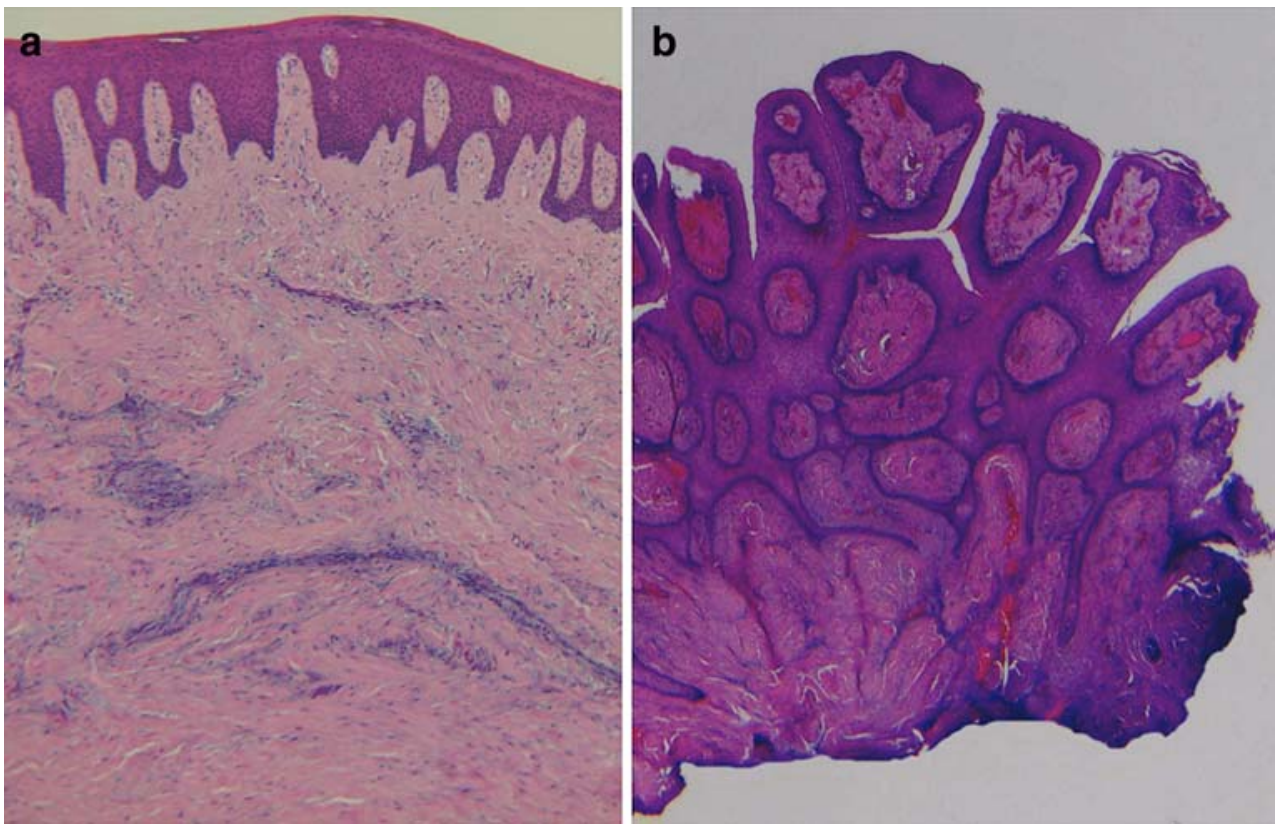
Fig. 4 Multipoint LOD score. The order of analyzed markers on chromosome $2 p$ is shown on the $X$-axis, and distances are given in centiMorgan. The $Y$ axis refers to the LOD score

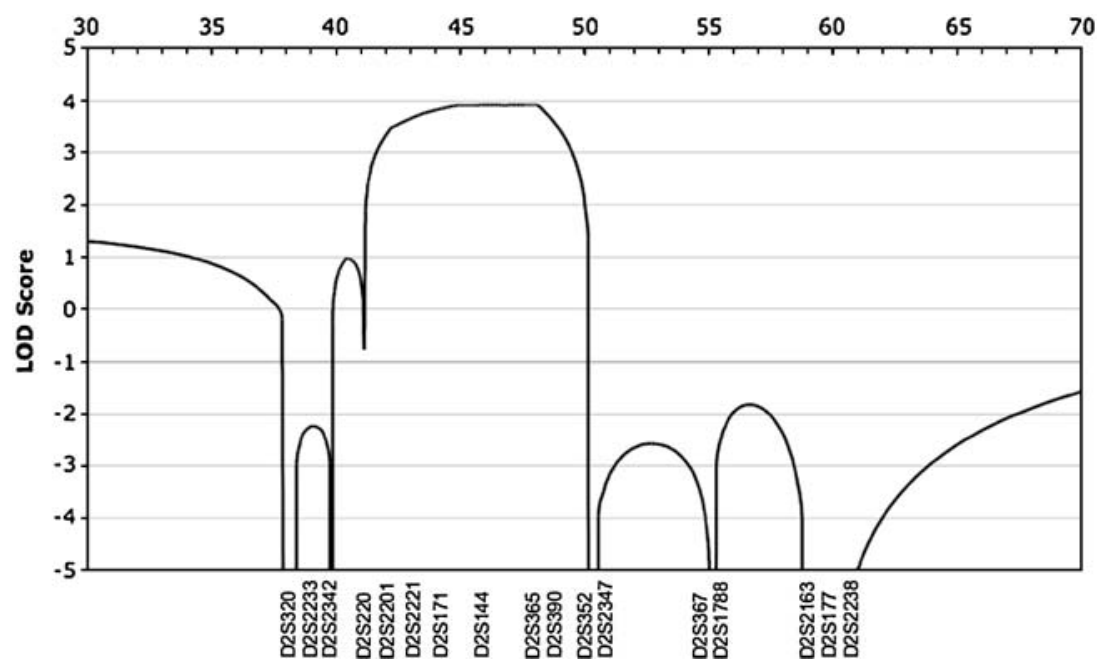

date region encoding for components of the RAS/MAPK pathway will be given priority regarding sequence analysis to identify the mutation causing HGF in the presented family. For example, RAB10 from the interval encodes a member of the RAS (see HRAS; OMIM 190020) superfamily of small GTPases. RAB proteins localize to exocytic and endocytic compartments and regulate intracellular vesicle trafficking; however, the particular function of RAB10 is unknown. Most genes from the candidate region are incompletely characterized such as GPN1, which encodes a guanosine triphosphatase enzyme that may play a role in DNA repair and may function in activation of transcription, and $A G B L 5$, a putative ATP/GTP binding protein. CIB4, encoding calcium and integrin binding family member 4 , may represent a further HGF candidate gene considering that calcium channel blockers can induce gingival hyperplasia. Candidates further include $P P P 1 C B$, encoding one of the three catalytic subunits of protein phosphatase 1, which is known to be involved in the regulation of cell division, glycogen metabolism, muscle

Table 1 Two-point linkage analysis between the HGF locus and chromosome $2 \mathrm{p}$ markers

\begin{tabular}{|c|c|c|c|c|c|c|c|c|c|c|}
\hline \multirow[t]{2}{*}{ Marker } & \multirow{2}{*}{$\begin{array}{l}\text { Genetic } \\
\text { Distance (cM) }\end{array}$} & \multicolumn{7}{|c|}{ LOD score at $\theta$} & \multirow[t]{2}{*}{$Z_{\max }$} & \multirow[t]{2}{*}{$\theta_{\max }$} \\
\hline & & 0.00 & 0.01 & 0.05 & 0.10 & 0.20 & 0.30 & 0.40 & & \\
\hline D2S320 & 38.33 & $-\infty$ & 1.04 & 1.53 & 1.58 & 1.44 & 1.22 & 1.01 & 1.58 & 0.09 \\
\hline D2S2233 & 39.93 & $-\infty$ & 0.78 & 1.29 & 1.36 & 1.24 & 1.06 & 0.87 & 1.36 & 0.10 \\
\hline D2S2342 & 40.47 & 0.95 & 0.93 & 0.84 & 0.74 & 0.56 & 0.41 & 0.29 & 0.95 & 0 \\
\hline D2S220 & 42.65 & -0.69 & 1.26 & 1.76 & 1.82 & 1.67 & 1.44 & 1.22 & 1.82 & 0.09 \\
\hline $\mathrm{D} 2 \mathrm{~S} 2201^{\mathrm{a}}$ & 41.57 & 1.83 & 1.79 & 1.64 & 1.45 & 1.13 & 0.87 & 0.66 & 1.83 & 0 \\
\hline D2S2221 & 44.09 & 2.19 & 2.15 & 1.99 & 1.80 & 1.46 & 1.16 & 0.92 & 2.19 & 0 \\
\hline D2S171 & 45.30 & 3.45 & 3.39 & 3.16 & 2.89 & 2.40 & 1.97 & 1.60 & 3.45 & 0 \\
\hline $\mathrm{D} 2 \mathrm{~S} 144$ & 45.30 & 3.42 & 3.36 & 3.13 & 2.86 & 2.37 & 1.95 & 1.59 & 3.42 & 0 \\
\hline D2S365 & 47.97 & 0.95 & 0.93 & 0.85 & 0.75 & 0.58 & 0.44 & 0.33 & 0.95 & 0 \\
\hline $\mathrm{D} 2 \mathrm{~S} 390$ & 48.50 & 3.31 & 3.25 & 3.03 & 2.77 & 2.30 & 1.89 & 1.54 & 3.31 & 0 \\
\hline D2S352 & 50.65 & $-\infty$ & -1.51 & -0.29 & 0.11 & 0.33 & 0.35 & 0.32 & 0.36 & 0.27 \\
\hline D2S2347 & 50.65 & $-\infty$ & -0.34 & 0.24 & 0.40 & 0.45 & 0.42 & 0.37 & 0.45 & 0.19 \\
\hline D2S367 & 54.96 & 0.95 & 0.93 & 0.88 & 0.82 & 0.69 & 0.59 & 0.49 & 0.95 & 0 \\
\hline D2S1788 & 55.51 & $-\infty$ & -0.92 & 0.27 & 0.62 & 0.76 & 0.71 & 0.61 & 0.76 & 0.20 \\
\hline D2S2163 & 59.36 & $-\infty$ & -0.61 & -0.02 & 0.15 & 0.23 & 0.22 & 0.19 & 0.23 & 0.23 \\
\hline D2S177 & 59.36 & $-\infty$ & -4.01 & -2.05 & -1.32 & -0.71 & -0.43 & -0.28 & -0.28 & 0.40 \\
\hline D2S2238 & 60.45 & $-\infty$ & -2.49 & -1.20 & -0.73 & -0.36 & -0.21 & -0.13 & -0.13 & 0.40 \\
\hline
\end{tabular}

${ }^{a}$ The physical map of chromosome 2 (NCBI draft sequence of the human genome, build 36.3) indicates that D2S2201 localizes centromeric to D2S220, contrasting with the Marshfield genetic map of chromosome 2. 
contractility, protein synthesis, and HIV-1 viral transcription, and the protein encoded by $F K B P 1 B$, a member of the immunophilin protein family, which play a role in immunoregulation and basic cellular processes involving protein folding and trafficking. FKBP1B is a cis-trans prolyl isomerase that binds the immunosuppressant FK506 (Tacrolimus), which can induce gingival overgrowth as a side-effect in treated patients $[16,17]$.

There are clinical and histological differences in the presented family as compared with SOS1-related gingival fibromatosis, which might reflect the different molecular causes of the disease. As frequently seen in HGF, an overall cell poor increase in gingival tissue with scarce blood vessels and islets with dense fibroblasts comparable to the findings in the Chinese family originally defining the GINGF3 locus [6] was noted in our patients contrasting with SOS1-related gingival fibromatosis, which features a higher increase in the number of fibroblasts compared with the increase in extracellular matrix [18]. The family presented here is also remarkable in that all patients consistently had an early onset of the disease, at the time of eruption of the primary teeth, also similar to the original Chinese GINGF3 family [6], together arguing for a clinically recognizable subtype of adHGF linked to GINGF3.

Identification of the genetic mutations involved in HGF would provide novel aids for disease diagnosis, uncover targets for novel treatment modalities, and improve our understanding of the molecular mechanisms underlying HGF and other fibrotic processes.

Acknowledgements This work was supported by grant no. P18470 from Fonds zur Förderung der Wissenschaftlichen Forschung in Österreich (FWF) to A.R.J..

Competing interest statement The authors declare that they have no competing financial interests.

Open Access This article is distributed under the terms of the Creative Commons Attribution Noncommercial License which permits any noncommercial use, distribution, and reproduction in any medium, provided the original author(s) and source are credited.

\section{References}

1. Hakkinen L, Csiszar A (2007) Hereditary gingival fibromatosis: characteristics and novel putative pathogenic mechanisms. J Dent Res 86:25-34
2. Hart TC, Pallos D, Bozzo L, Almeida OP, Marazita ML, O'Connell JR, Cortelli JR (2000) Evidence of genetic heterogeneity for hereditary gingival fibromatosis. J Dent Res 79:1758-1764

3. Goldblatt J, Singer SL (1992) Autosomal recessive gingival fibromatosis with distinctive facies. Clin Genet 42:306-308

4. Hart TC, Pallos D, Bowden DW, Bolyard J, Pettenati MJ, Cortelli JR (1998) Genetic linkage of hereditary gingival fibromatosis to chromosome 2p21. Am J Hum Genet 62:876-883

5. Xiao S, Wang X, Qu B, Yang M, Liu G, Bu L, Wang Y, Zhu L, Lei $\mathrm{H}, \mathrm{Hu} \mathrm{L}$, Zhang $\mathrm{X}$, Liu J, Zhao G, Kong X (2000) Refinement of the locus for autosomal dominant hereditary gingival fibromatosis (GINGF) to a $3.8-\mathrm{cM}$ region on $2 \mathrm{p} 21$. Genomics 68:247-252

6. Ye X, Shi L, Cheng Y, Peng Q, Huang S, Liu J, Huang M, Peng B, Bian Z (2005) A novel locus for autosomal dominant hereditary gingival fibromatosis, GINGF3, maps to chromosome 2p22.3-p23.3. Clin Genet 68:239-244

7. Xiao S, Bu L, Zhu L, Zheng G, Yang M, Qian M, Hu L, Liu J, Zhao G, Kong X (2001) A new locus for hereditary gingival fibromatosis (GINGF2) maps to 5q13-q22. Genomics 74:180185

8. Shashi V, Pallos D, Pettenati MJ, Cortelli JR, Fryns JP, von Kap-Herr C, Hart TC (1999) Genetic heterogeneity of gingival fibromatosis on chromosome 2p. J Med Genet 36:683-686

9. Zhu Y, Zhang W, Huo Z, Zhang Y, Xia Y, Li B, Kong X, Hu L (2007) A novel locus for maternally inherited human gingival fibromatosis at chromosome 11p15. Hum Genet 121:113-123

10. Gudbjartsson DF, Thorvaldsson T, Kong A, Gunnarsson G, Ingolfsdottir A (2005) Allegro version 2. Nat Genet 37:10151016

11. Seelow D, Schwarz JM, Schuelke M (2008) GeneDistillerdistilling candidate genes from linkage intervals. PLoS ONE 3: e3874

12. Hart TC, Zhang Y, Gorry MC, Hart PS, Cooper M, Marazita ML, Marks JM, Cortelli JR, Pallos D (2002) A mutation in the SOS1 gene causes hereditary gingival fibromatosis type 1. Am J Hum Genet 70:943-954

13. Jang SI, Lee EJ, Hart PS, Ramaswami M, Pallos D, Hart TC (2007) Germ line gain of function with SOS1 mutation in hereditary gingival fibromatosis. J Biol Chem 282:20245-20255

14. Digilio MC, Sarkozy A, Capolino R, Chiarini Testa MB, Esposito G, de Zorzi A, Cutrera R, Marino B, Dallapiccola B (2008) Costello syndrome: clinical diagnosis in the first year of life. Eur J Pediatr 167:621-628

15. Sun M, Li N, Dong W, Chen Z, Liu Q, Xu Y, He G et al (2009) Copy-number mutations on chromosome 17q24.2-q24.3 in congenital generalized hypertrichosis terminalis with or without gingival hyperplasia. Am J Hum Genet 84:807-813

16. Ellis JS, Seymour RA, Taylor JJ, Thomason JM (2004) Prevalence of gingival overgrowth in transplant patients immunosuppressed with tacrolimus. J Clin Periodontol 31:126-131

17. Lima RB, Benini V, Sens YA (2008) Gingival overgrowth in renal transplant recipients: a study concerning prevalence, severity, periodontal, and predisposing factors. Transplant Proc 40:14251428

18. Lee EJ, Jang SI, Pallos D, Kather J, Hart TC (2006) Characterization of fibroblasts with Son of Sevenless-1 mutation. J Dent Res 85:1050-1055 